\title{
Bacteriostatic versus bactericidal activity of ciprofloxacin in Escherichia coli assessed by flow cytometry using a novel far-red dye
}

\author{
Filomena Silva, Olga Lourenço, João A Queiroz and Fernanda C Domingues
}

\begin{abstract}
As common microbiological methods for the assessment of bacteriostatic or bactericidal activities are very time-consuming, in this work we describe that the use of a novel far-red fluorescent stain, Vybrant DyeCycle Ruby (DCR) for the flow cytometric analysis of fluoroquinolone (ciprofloxacin) bacteriostatic and bactericidal activities in Escherichia coli proved to be specific for bacterial DNA and, after ciprofloxacin exposure, DNA distribution analysis was achieved using a 7.5 $\mu \mathrm{m}$ DCR concentration to stain $5 \times 10^{5}$ ethanol-fixed bacterial cells. The analysis of the bacterial DNA histograms obtained from the ciprofloxacin concentrations tested, enabled the distinction between ciprofloxacin bacteriostatic and bactericidal activities.
\end{abstract} The Journal of Antibiotics (2011) 64, 321-325; doi:10.1038/ja.2011.5; published online 16 February 2011

Keywords: bacterial DNA; ciprofloxacin; flow cytometry; Vybrant DyeCycle Ruby

\section{INTRODUCTION}

In clinical practice, optimal dosing is a critical component of antibiotic therapy, regardless of the type or class of agents employed. ${ }^{1}$ In patients with serious bacterial infections, the use of maximum tolerable antibiotic doses improves therapeutic outcomes, and may prevent the development of resistance. ${ }^{2}$ Bactericidal rather than bacteriostatic agents may be preferred, as these agents may limit the development of resistance. ${ }^{3}$ However, even when bactericidal agents are used, the administered dose must be sufficient to achieve bactericidal activity, ${ }^{1}$ because with some antibacterials (for example, aminoglycosides and fluoroquinolones) bacterial killing is concentrationdependent. Therefore, it is important to develop fast and simple methodologies to assess whether the antibiotic dose administered produces bactericidal or bacteriostatic activities against the pathogens.

The use of flow cytometry as an important tool in bacteriology is emerging. ${ }^{4}$ Flow cytometric measurement of DNA content may be important in understanding and quantifying bacterial heterogeneity. Many studies involving bacterial DNA content analysis have been performed with UV-excitable, DNA-specific fluorescent stains, such as Hoechst dyes and 4',6-diamidino-2-phenylindole (DAPI). However, the use of these fluorescent stains required the use of UV lasers, and the flow cytometers that can accommodate UV excitation remain uncommon and are considerably more expensive than conventional cytometers. ${ }^{5}$ In order to circumvent this, new fluorescent stains excitable with violet $(395-415 \mathrm{~nm})$, blue $(488 \mathrm{~nm})$ and red $(635 \mathrm{~nm})$ lasers have been developed. Examples of such fluorescent stains are the Vybrant DyeCycle series (Molecular Probes, Invitrogen, Carlsbad, CA, USA) and DRAQ5 (Biostatus Limited, Leicestershire, UK). Some of these dyes have been successfully used to assess intracellular DNA distribution in live ${ }^{6-8}$ and fixed cells. ${ }^{9}$ Recently, a Vybrant DyeCycle stain was developed, Vybrant DyeCycle Ruby (DCR), which has the same features of the other related stains and can be used with any excitation source from 488 to $690 \mathrm{~nm}$ with fluorescence emission in the near-infrared region.

Fluoroquinolones are broad-spectrum antibacterial agents that are extensively used for treatment of infections. ${ }^{10}$ Depending on their concentration, fluoroquinolones may exhibit bacteriostatic or bactericidal activities. ${ }^{11}$ For instance, ciprofloxacin, a fluoroquinolone, exhibits a bacteriostatic activity when the replication of DNA is inhibited by inhibiting DNA gyrase and a bactericidal activity caused by bacterial DNA fragmentation. Therefore, this work reports the applicability of a novel far-red dye, DCR, for the rapid and easy evaluation of bactericidal and bacteriostatic activities of ciprofloxacin. This analysis is performed by the evaluation of the dye effects in the DNA content of E. coli by flow cytometry.

\section{MATERIALS AND METHODS}

Bacterial strains and antibiotics

The bacterial strain used was Escherichia coli ATCC 25922. Ciprofloxacin (Sigma-Aldrich, St Louis, MO, USA) stock solutions were prepared at $1 \mathrm{mg} \mathrm{ml}^{-1}$ in $0.1 \mathrm{~N} \mathrm{HCl}$, sterilized by filtration $(0.22 \mu \mathrm{m}$ pore-size filter $)$, dispensed in $0.5 \mathrm{ml}$ aliquots and frozen at $-80^{\circ} \mathrm{C}$ until use.

Ciprofloxacin susceptibility testing

The in vitro activity of ciprofloxacin was determined by the broth microdilution method, according to the Clinical and Laboratory Standards Institute 
(M7-A6), ${ }^{12}$ with cation-adjusted Müeller-Hinton broth. Microtiter plates containing serial dilutions of ciprofloxacin ranging from 4 to $0.001 \mu \mathrm{g} \mathrm{m}^{-1}$ were inoculated with E. coli to yield approximately $5 \times 10^{5}$ cells per $\mathrm{ml}$ in a $100 \mu \mathrm{l}$ final volume. The plates were incubated for $18 \mathrm{~h}$ at $37^{\circ} \mathrm{C}$. The minimal inhibitory concentration (MIC) was defined as the lowest concentration of ciprofloxacin that prevented visible growth.

\section{Time-kill studies}

Time-kill assays were performed by the broth macrodilution method, according to the Clinical and Laboratory Standards Institute guidelines. ${ }^{13}$ For the concentration-dependent killing curve studies, various multiples of the MICs $(0.5,1,2$ and 4$)$ were used to detect differences in killing. Shake flasks $(250 \mathrm{ml})$ containing $50 \mathrm{ml}$ of cation-adjusted Müeller-Hinton broth with the appropriate ciprofloxacin concentrations were inoculated with $50 \mathrm{ml}$ of the exponentially grown $E$. coli cells, to yield a final concentration of approximately $1 \times 10^{6}$ cells per ml. Flasks were incubated at $37^{\circ} \mathrm{C}$, and aliquots were removed at $0,2,4,6$ and $24 \mathrm{~h}$ for the determination of viable counts. Serial dilutions were prepared in sterile $0.85 \%$ sodium chloride solution and plated according to the method previously described. ${ }^{14}$ The plates were incubated at $37^{\circ} \mathrm{C}$ for 18 to $22 \mathrm{~h}$, and the number of colonies was determined. The detection level by this plating method was $1 \times 10^{2} \mathrm{CFU} \mathrm{ml}^{-1}$.

Killing curves were constructed by plotting the $\log _{10} \mathrm{CFU} \mathrm{m}{ }^{-1}$ versus time over $24 \mathrm{~h}$, and the change in bacterial concentration was determined. Bactericidal activity was defined as a reduction of $99.9 \%(\geqslant 3 \log 10)$ of the total number of $\mathrm{CFU} \mathrm{ml}{ }^{-1}$ in the original inoculum. ${ }^{15}$ Bacteriostatic activity was defined as maintenance of the original inoculum concentration or a reduction of less than $99.9 \%(<3 \log 10)$ of the total number of $\mathrm{CFU} \mathrm{ml}^{-1}$ in the original inoculum. ${ }^{16}$

\section{Ciprofloxacin exposure and cell recovery for flow cytometric and microscopic analysis}

A pre-culture was performed by growing E. coli cells in Brain Heart Infusion broth until mid-exponential phase of growth was reached. Cells taken from the pre-culture $\left(1 \times 10^{6}\right.$ cells per $\left.\mathrm{ml}\right)$ were incubated in cation-adjusted MüellerHinton broth with ciprofloxacin at $0.5,1,2$ and 4 times the MIC value. As a control of bacterial growth, bacterial cultures were grown without the addition of ciprofloxacin. All cultures were incubated in the same conditions described previously for the time-kill studies. Three 1-ml samples were removed from the control culture prior to incubation, and further three 1-ml samples were removed from cultures after 2, 4 and $6 \mathrm{~h}$ of incubation. Samples were centrifuged at $10,000 \mathrm{~g}$ and the pellet was washed with phosphate-buffered saline (PBS) buffer ( $\mathrm{pH}$ 7.4) once and resuspended in $2 \mathrm{ml}$ Müeller-Hinton broth for plate counts and microscopic analysis. In order to improve the ability to resolve ploidy, cell pellet was also resuspended in Müeller-Hinton broth containing $300 \mu \mathrm{g} \mathrm{ml}^{-1}$ rifampicin and $10 \mu \mathrm{g} \mathrm{ml}^{-1}$ cephalexin, as previously described. ${ }^{9}$ After this treatment, bacterial cells were ethanol-fixed as described below.

\section{Bacterial fixation and staining}

A $700 \mu \mathrm{l}$ volume of ice-cold absolute ethanol was rapidly added to the $300 \mu \mathrm{l}$ of recovered cells after ciprofloxacin exposure, as described above. This suspension was mixed by rapid pipetting to prevent cell clumping. The samples were then incubated at $4{ }^{\circ} \mathrm{C}$ for $30 \mathrm{~min}$ for fixation. Fixed cells were stored at $-20{ }^{\circ} \mathrm{C}$ until they were stained, and analyzed on the flow cytometer. After optimization of both incubation time and DCR concentration, $5 \times 10^{5}$ cells per ml were washed once with PBS buffer (pH 7.4), incubated with $7.5 \mu \mathrm{m}$ DCR in PBS buffer (pH 7.4) at $37^{\circ} \mathrm{C}$ for $30 \mathrm{~min}$ in the dark and washed once in the same buffer before the flow cytometric analysis.

As a control for DCR bacterial DNA specificity, ethanol-fixed cells were incubated with DNase and/or RNase as described. ${ }^{9}$

\section{Flow cytometry}

DCR (Molecular Probes, Invitrogen, part of Life Technologies, Carlsbad, CA, USA) stock solution $(2.5 \mathrm{mM})$ in dimethylsulphoxide was stored at $-20^{\circ} \mathrm{C}$. Bacterial samples were analyzed on a BD Biosciences FACSCalibur (BD Biosciences, San Jose, CA, USA). Acquisition was performed with CellQuest Pro software (BD Biosciences) and based on light-scatter and fluorescence signals resulting from $15 \mathrm{~mW}$ laser illumination at 488 and $635 \mathrm{~nm}$. Light- scatter measurements were acquired logarithmically, while fluorescence signals were acquired linearly. DCR fluorescence signal was screened with a FL-4 $(661 \mathrm{~nm})$ bandpass filter. Threshold levels were set on SSC to eliminate noise or particles (of cellular debris) much smaller than intact cells. Bacterial cells were gated according to FSC/SSC parameters. Daily calibration was performed using CaliBRITE beads (BD Biosciences), according to the manufacturer instructions. Sample acquisition was operated at low flow rate setting $(12 \mu \mathrm{l}$ sample per minute). A total of 30,000 events were acquired for each sample. Data analysis was performed using FCS Express version 3 Research Edition (De Novo Software, Los Angeles, CA, USA).

\section{Epifluorescence microscopy}

Bacterial cell suspensions, after ciprofloxacin exposure and recovery, were deposited on poly(D-lysine)-coated coverslips previously placed in the wells of a 48 -well polystyrene plate. The plates were incubated at $37^{\circ} \mathrm{C}$ for $45 \mathrm{~min}$ to allow the adhesion of E. coli cells onto the coverslips. The plates were then washed with PBS buffer and fixed for $10 \mathrm{~min}$ at room temperature with $4 \%$ paraformaldehyde solution. The plates were washed with PBS buffer and permeabilized for $5 \mathrm{~min}$ at room temperature with $1 \%$ Triton X-100 solution. The plates were washed with PBS buffer and a $7.5 \mu \mathrm{m}$ DCR solution was added to the wells. The plates were then incubated at $37^{\circ} \mathrm{C}$ for $30 \mathrm{~min}$. The plates were washed with PBS buffer, and a $6 \mu \mathrm{g} \mathrm{ml}^{-1}$ fluorescein 5(6)-isothiocyanate (Sigma-Aldrich) solution was added to the wells in order to stain whole cells. The plates were then incubated at $37^{\circ} \mathrm{C}$ for $30 \mathrm{~min}$; afterwards, cells were washed twice, and the coverslips were mounted onto glass slides with DAKO (Dako, Glostrup, Denmark) mounting medium. These slides were then examined under an epifluorescence microscope $(\times 100)$ in a Zeiss AX10 microscope using the Axio Vision Real 4.6 Software (Carl Zeiss MicroImaging GmbH, Jena, Germany).

\section{RESULTS}

\section{DCR specificity}

In order to verify DCR specificity for bacterial DNA, fixed cells were subjected to treatments with DNase, RNase and with both these enzymes before DCR labeling. The analysis of light-scatter parameters showed that there were no significant changes in particle size distribution after enzyme treatments (data not shown). DNase treatment (Figure $1 \mathrm{~b}$ ) yielded a very similar histogram to that obtained after the treatment with both enzymes (Figure 1c). Both of these histograms exhibited a slightly higher FL4 channel fluorescence comparing with the non-stained population (Figure 1a). RNase treatment yielded a very similar FL4 channel fluorescence distribution when compared with E. coli cells without treatment (Figures $1 \mathrm{~d}$ and e). The fact that this stain is DNA-specific is further supported by the microscopic evaluation of DCR- and fluorescein 5(6)-isothiocyanate-stained E. coli cells, where DCR-stained E. coli chromosomes could be observed (Figure 1f).

\section{Bacteriostatic versus bactericidal activity of ciprofloxacin}

The ciprofloxacin MIC value obtained for Escherichia coli ATCC 25922 was $0.008 \mu \mathrm{g} \mathrm{ml}^{-1}$. In order to assess if the concentrations used resulted in either a bacteriostatic or bactericidal effect, a time-kill study of bacterial cells exposed to ciprofloxacin concentrations ranging from 0.5 to 4 times the MIC value over $24 \mathrm{~h}$ incubation at $37^{\circ} \mathrm{C}$ was performed (Figure 2). As it is known that bacterial growth phase can influence susceptibility profiles, ${ }^{17}$ a pre-culture was performed to obtain a homogeneous population of cells in an active state as confirmed by propidium iodide staining (data not shown).

Time-kill curves showed that bacterial killing is concentrationdependent, for the rate of killing increases with progressively higher antibacterial concentrations. Incubation of $E$. coli cells with a ciprofloxacin concentration of $0.5 \times$ MIC did not result in a decreased bacterial cell count (Figure 2). For ciprofloxacin concentration of one time the MIC $\left(0.008 \mu \mathrm{g} \mathrm{ml}^{-1}\right)$, the reduction in bacterial cell counts 

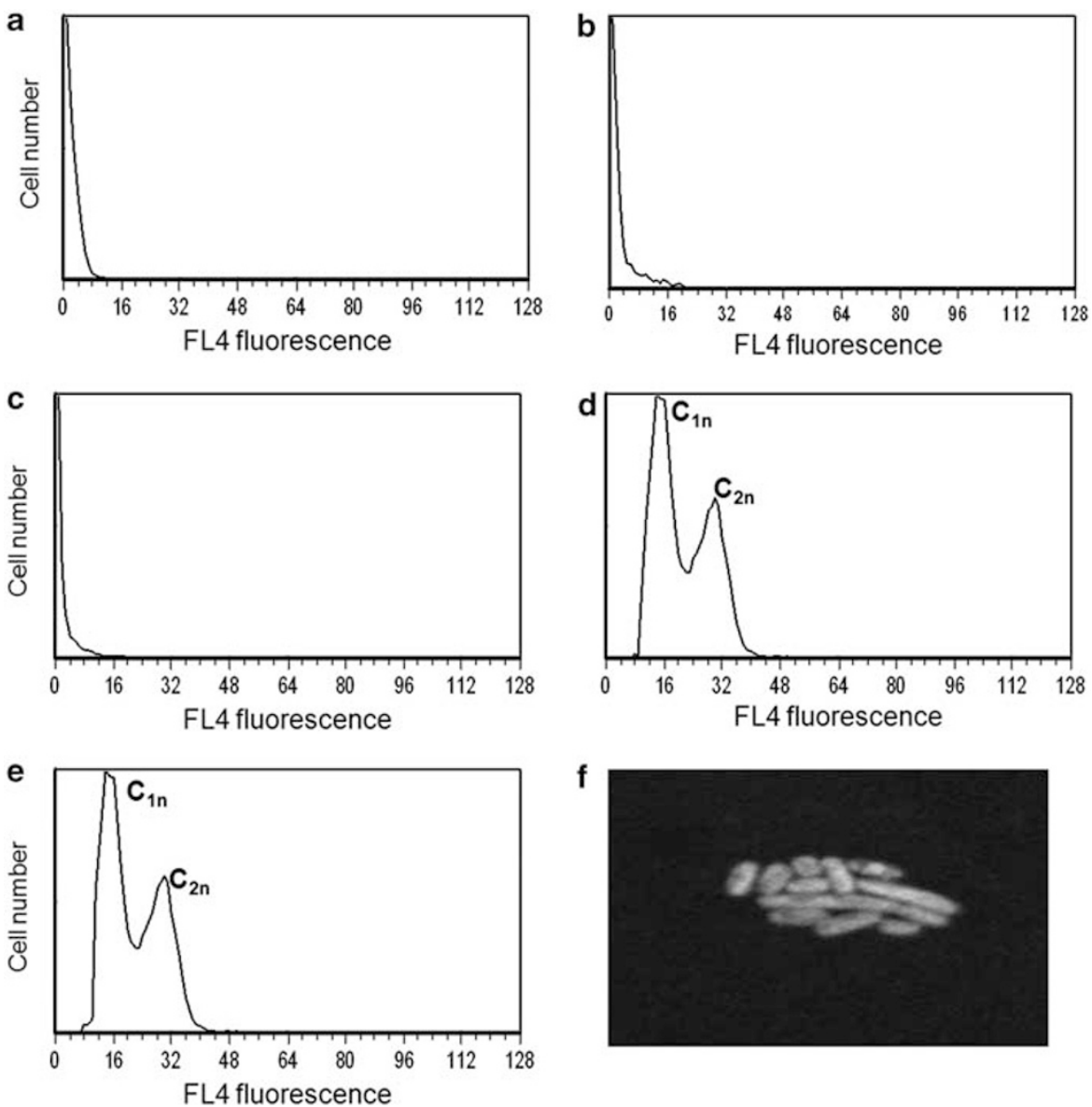

Figure 1 Effect of enzyme digestion on the DyeCycle Ruby (DCR) staining of fixed E. coli cells. (a) Untreated suspension without staining, (b) stained cell suspension after DNase treatment, (c) stained cell suspension after DNase and RNase treatment, (d) stained cell suspension after RNase treatment and (e) untreated and stained cell suspension; (f) epifluorescence microscopy images of $E$. coli cells immobilized on poly(D-lysine)-coated coverslips and incubated with DCR (red) and fluorescein 5(6)-isothiocyanate (green), as described in Materials and methods. A total of 20,000 events were collected for the flow cytometric analysis. A full color version of this figure is available at The Journal of Antibiotics journal online.

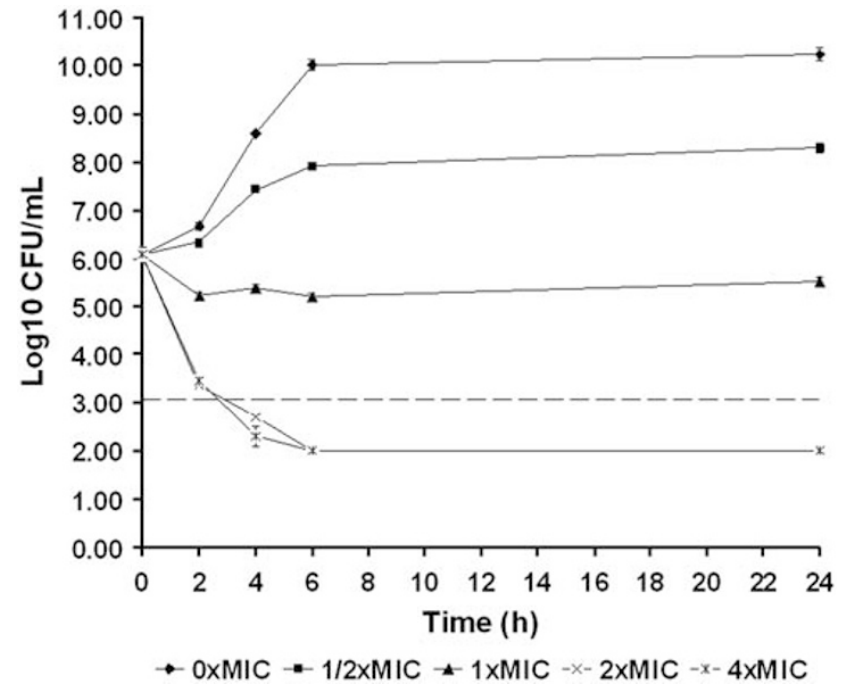

Figure 2 Viable counts for cultures of $E$. coli. Results are shown as the mean \pm s.d. values for three experiments of control cultures $(\downarrow)$ and cultures treated at $37^{\circ} \mathrm{C}$ during $24 \mathrm{~h}$, with ciprofloxacin concentrations corresponding to $0.5(\boldsymbol{\square}), 1(\boldsymbol{\Delta}), 2(\times)$ and $4\left(^{*}\right)$ times the MIC value determined. was less than $3 \log _{10} \mathrm{CFU} \mathrm{ml}^{-1}$, indicative of a bacteriostatic effect. A bactericidal effect was obtained when $E$. coli cells were incubated with ciprofloxacin concentrations of two and four times the MIC $\left(0.016\right.$ and $0.032 \mu \mathrm{g} \mathrm{ml}^{-1}$, respectively) as can be seen by a reduction in bacterial cell counts of $4.0 \log _{10} \mathrm{CFU} \mathrm{ml} \mathrm{m}^{-1}$ (Figure 2).

As bacteriostatic and bactericidal activities of ciprofloxacin were obtained for ciprofloxacin concentrations equaling one time and four times the MIC, respectively (Figure 2), those concentrations were chosen to perform the flow cytometric studies.

DNA histograms of E. coli cells not exposed to ciprofloxacin (Figure 3a) showed two peaks corresponding to one or two chromosome equivalents, and a ridge connecting the two peaks, which is indicative of actively growing cells. At $2 \mathrm{~h}$ of incubation, the majority of cells contained one chromosome equivalent; at $4 \mathrm{~h}$, there is an increase of the peak corresponding to cells with two chromosome equivalents, which suffers a reduction at $6 \mathrm{~h}$, where most cells contained one chromosome equivalent, as can be seen in the fluorescence microscopy image (Figure 4a). When analyzing DNA histograms of cells exposed to ciprofloxacin at one time the MIC (bacteriostatic effect) (Figure 3b), there is a decrease in peak resolution throughout incubation time and all DNA histograms are very similar, with cells containing one or two chromosome equivalents. These cells 
a

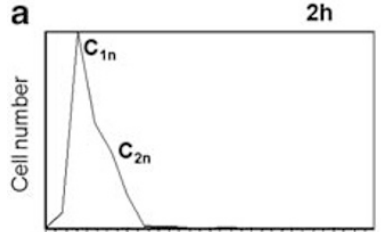

FL4 fluorescence

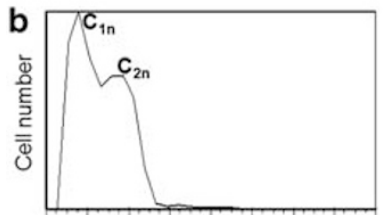

FL4 fluorescence

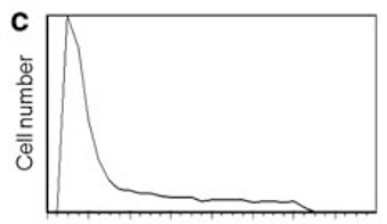

FL4 fluorescence

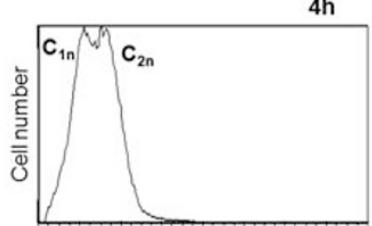

FL4 fluorescence

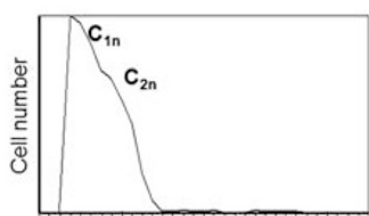

FL4 fluorescence

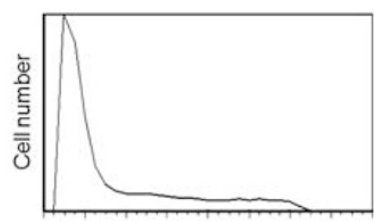

FL4 fluorescence

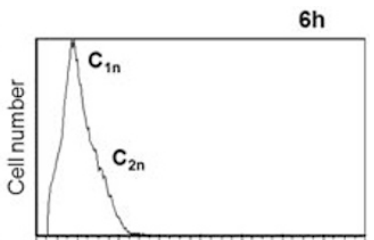

FL4 fluorescence

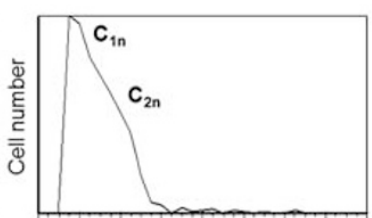

FL4 fluorescence

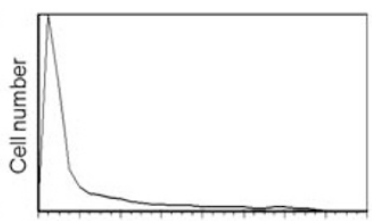

FL4 fluorescence

Figure 3 Effect of ciprofloxacin concentration on E. coli DNA content. Histograms show the distribution of DyeCycle Ruby fluorescence intensity (FL4 channel) in a linear scale using E. coli cultures (a) not treated or exposed to a ciprofloxacin concentration of: (b) one time the MIC and (c) four times the MIC. A total of 30,000 events were collected for this analysis.
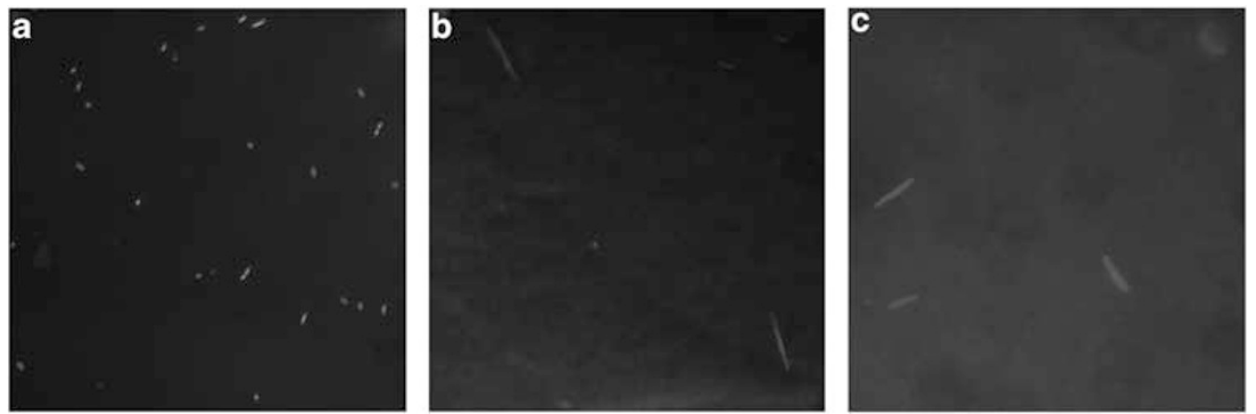

Figure 4 Epifluorescence microscopy images of $E$. coli cells after $6 \mathrm{~h}$ of incubation. (a) Control, (b) cells exposed to a ciprofloxacin concentration of one time the MIC and (c) four times the MIC. Cells were immobilized on poly(D-lysine)-coated coverslips and incubated with DyeCycle Ruby (red) and fluorescein 5(6)isothiocyanate (green), as described in Materials and methods. A full color version of this figure is available at The Journal of Antibiotics journal online.

exhibited a high filamentous structure where the chromosomes can be observed (Figure $4 \mathrm{~b}$ ). Cells exposed to a ciprofloxacin concentration of four times the MIC (bactericidal effect) (Figure 3c) exhibited DNA histograms with only one peak, with lower fluorescence intensities and a broadening of the histogram for higher fluorescence intensities. Because of this fact, the number of chromosome equivalents cannot be accurately determined. These findings are further supported by the microscopic visualization of the cells (Figure 4c) in which a filamentous structure can be observed, and because of the low fluorescence intensities the chromosomes cannot be fully distinguished.

\section{DISCUSSION}

As fluorescent stains with the Vybrant DyeCycle series have proven to be effective in staining live eukaryotic cells ${ }^{18}$ and fixing prokaryotic cells, such as those of the Gram-negative bacterium Caulobacter crescentus, ${ }^{19}$ the present work investigates the ability of DCR to stain bacterial DNA and further evaluates ciprofloxacin activity in the Gram-negative bacterium E. coli.

As one of the major characteristics of these fluorescent stains is that they bound selectively to eukaryotic DNA, it was important to clarify if DCR was also specific for bacterial DNA. DCR proved to be specific for bacterial DNA, as DNase treatments yielded a fluorescence distribution similar to that without DCR labeling, while RNase treatment yielded a fluorescence distribution similar to that with DCR labeling.

The dose-dependent bactericidal activity of ciprofloxacin is in accordance with previous studies. ${ }^{20}$ The marked decrease in bacterial viability could be explained by the fact that, in Gram-negative bacteria, ciprofloxacin is directed at DNA gyrase, which results in rapid blockage of DNA synthesis. ${ }^{11}$ Despite this decrease in bacterial viability, flow cytometric cell counts were not affected (data not shown), which is in agreement with the results obtained by other authors. ${ }^{21}$

As ciprofloxacin exhibited bacteriostatic and bactericidal activities at the concentrations tested, the next step was to develop a flow cytometric method that allowed the assessment of ciprofloxacin activity.

Previous studies reported that ciprofloxacin does not have a direct action on bacterial cell membrane integrity even at high concentrations. $^{21,22}$ Therefore, propidium iodide was not an effective indicator of ciprofloxacin susceptibility, and was not considered for this work. 
Another approach described in the literature to evaluate ciprofloxacin exposure consisted of the evaluation of membrane potential ${ }^{21,22}$ using bis-oxonol. As ciprofloxacin causes membrane depolarization at high concentrations, this could be seen as a good method to evaluate ciprofloxacin susceptibility. However, membrane potential is reversible, and other studies have shown that depolarized cells are able to grow when placed in fresh medium; ${ }^{23}$ thus, the percentage of stained cells will not reflect bacterial viability.

It is known that ciprofloxacin has a direct action on chromosomal DNA replication and fragmentation; ${ }^{24}$ hence, we intended to evaluate its activity by flow cytometric analysis of intracellular of DNA content using DCR.

The results obtained clearly showed two distinct DNA patterns related to two distinct ciprofloxacin activities: bacteriostatic and bactericidal. Ciprofloxacin bacteriostatic activity is due to an inhibition of DNA synthesis and cell growth. ${ }^{25}$ E. coli DNA histograms corresponding to ciprofloxacin bacteriostatic activity showed a similar profile and fluorescence intensities over the $6 \mathrm{~h}$ of incubation time. All DNA histograms showed two peaks corresponding to one and two chromosome equivalents, as a result the fluorescence intensity values obtained. The resolution of the histograms decreased with time, indicating that rifampicin and cefalexin treatments are no longer effective, perhaps because of ciprofloxacin action in bacterial DNA. These cells exhibited a highly filamentous morphology, which is in agreement with previous studies. ${ }^{21}$ In these filamentous cells, one or two chromosomes can be observed by fluorescence microscopy, further supporting the results obtained by flow cytometry.

Ciprofloxacin bactericidal activity is thought to be related to the release of free DNA ends from the DNA gyrase-quinolone complexes, ${ }^{25}$ leading to chromosomal DNA fragmentation. ${ }^{24}$ E. coli DNA histograms corresponding to ciprofloxacin bactericidal concentration showed similar fluorescence intensities throughout the $6 \mathrm{~h}$ of incubation. Because of the high ciprofloxacin concentrations used, cellular repair mechanisms were not able to increase DNA synthesis ${ }^{26}$ and, therefore, a complete inhibition of DNA synthesis is observed. Furthermore, the lower fluorescence intensities obtained and higher fluorescence broadening without visible peaks could be related to DNA fragmentation, ${ }^{27}$ because DNA fragments have a compromised ability to be stained with intercalating dyes, ${ }^{24}$ such as DCR, resulting in lower relative DCR fluorescence. These results were further supported by microscopic images, which showed a low staining level and the inability of visualizing chromosomes. These cells have a filamentous morphology with larger cell size than that of the control culture, but with smaller cell size when compared with cells incubated with one time the MIC (bacteriostatic effect). This cell filamentation as a result of ciprofloxacin exposure is well documented ${ }^{21,28}$ and can be seen as an indicative of the induction of SOS response. ${ }^{29}$

Because of the characteristics of the DNA-selective fluorescent stain used, DCR, it was possible to verify the applicability of this fluorescent stain to analyze bacterial DNA patterns for the purpose of studying ciprofloxacin activity. This flow cytometric analysis can also be applied to other antibiotics that are known to have different effects on bacterial DNA, such as aminoglycosides.

\section{ACKNOWLEDGEMENTS}

This work was supported by FCT, the Portuguese Foundation for Science and Technology (PTDC/EQU-EQU/65492/2006). Filomena Silva acknowledges a $\mathrm{PhD}$ fellowship SFRH/BD/41521/2007 from FCT. We would like to thank Molecular Probes, Invitrogen, part of Life Technologies (Carlsbad, California, USA) for kindly providing Vybrant DyeCycle Ruby stock solution. We would also like to acknowledge A. Martinho for her help with the preparation and visualization of samples for the fluorescence microscopy study.

1 Pankey, G. A. \& Sabath, L. D. Clinical relevance of bacteriostatic versus bactericidal mechanisms of action in the treatment of Gram-positive bacterial infections. Clin. Infect. Dis. 38, 864-870 (2004).

2 Drlica, K. \& Zhao, X. Mutant selection window hypothesis updated. Clin. Infect. Dis. 44, 681-688 (2007).

3 Stratton, C. W. Dead bugs don't mutate: susceptibility issues in the emergence of bacterial resistance. Emerg. Infect. Dis. 9, 10-16 (2003).

4 Barbesti, S. et al. Two and three-color fluorescence flow cytometric analysis of immunoidentified viable bacteria. Cytometry 40, 214-218 (2000).

5 Telford, W. G., Bradford, J., Godfrey, W., Robey, R. W. \& Bates, S. E. Side population analysis using a violet-excited cell-permeable DNA binding dye. Stem Cells 25, 1029-1036 (2007).

6 Mori, T. et al. A stable human progesterone receptor expressing HeLa reporter cell line as a tool in chemical evaluation at the different cell-cycle phases. Toxicol. Lett. 186, 123-129 (2009).

7 Mukherjee, C., Majumder, S. \& Lohia, A. Inter-cellular variation in DNA content of Entamoeba histolytica originates from temporal and spatial uncoupling of cytokinesis from the nuclear cycle. PLoS Negl. Trop. Dis. 3, e409 (2009).

8 Zemans, R. L. et al. A novel method for long term bone marrow culture and genetic modification of murine neutrophils via retroviral transduction. J. Immunol. Methods 340, 102-115 (2009).

9 Silva, F. et al. The use of DRAQ5 to monitor intracellular DNA in Escherichia coli by flow cytometry. J. Fluoresc. 20, 907-914 (2010).

10 Demain, A. L. \& Sanchez, S. Microbial drug discovery: 80 years of progress. J. Antibiot. (Tokyo) 62, 5-16 (2009).

11 Drlica, K. Mechanism of fluoroquinolone action. Curr. Opin. Microbiol. 2, 504-508 (1999).

12 NCCLS. Methods for dilution antimicrobial susceptibility tests for bacteria that grow aerobically — sixth edition: approved standard M7-A6. National Committee for Clinical Laboratory Standards, Wayne, PA (2003).

13 NCCLS. Methods for determining bactericidal activity of antimicrobial agents; approved guidelines, M26-A, vol. 19. National Committee for Clinical Laboratory Standards, Wayne, PA (1999).

14 Chen, C. Y., Nace, G. W. \& Irwin, P. L. A $6 \times 6$ drop plate method for simultaneous colony counting and MPN enumeration of Campylobacter jejuni, Listeria monocytogenes, and Escherichia coli. J. Microbiol. Methods 55, 475-479 (2003).

15 NCCLS. Methods for determining bactericidal activity of antimicrobial agents: approved guideline M26-A. National Committee for Clinical Laboratory Standards, Wayne, PA (1999).

16 Petersen, P. J., Jones, C. H. \& Bradford, P. A. In vitro antibacterial activities of tigecycline and comparative agents by time-kill kinetic studies in fresh Mueller-Hinton broth. Diagn. Microbiol. Infect. Dis. 59, 347-349 (2007).

17 Paulo, L., Ferreira, S., Gallardo, E., Queiroz, J. A. \& Domingues, F. Antimicrobial activity and effects of resveratrol on human pathogenic bacteria. World J Microbiol Biotechnol 26, 1533-1538 (2010).

18 Mathew, G.. et al. ABCG2-mediated DyeCycle Violet efflux defined side population in benign and malignant prostate. Cell Cycle 8, 1053-1061 (2009).

19 Lesley, J. A. \& Shapiro, L. SpoT regulates DnaA stability and initiation of DNA replication in carbon-starved Caulobacter crescentus. J. Bacteriol. 190, 6867-6880 (2008).

20 Spangler, S. K., Jacobs, M. R. \& Appelbaum, P. C. Time-kill study of the activity of trovafloxacin compared with ciprofloxacin, sparfloxacin, metronidazole, cefoxitin, piperacillin and piperacillin/tazobactam against six anaerobes. J. Antimicrob. Chemother. 39 (Suppl B), 23-27 (1997).

21 Mason, D. J., Power, E. G., Talsania, H., Phillips, I. \& Gant, V. A. Antibacterial action of ciprofloxacin. Antimicrob. Agents Chemother. 39, 2752-2758 (1995).

22 Wickens, H. J., Pinney, R. J., Mason, D. J. \& Gant, V. A. Flow cytometric investigation of filamentation, membrane patency, and membrane potential in Escherichia coli following ciprofloxacin exposure. Antimicrob. Agents Chemother. 44, 682-687 (2000).

23 Nebe-von-Caron, G., Stephens, P. J., Hewitt, C. J., Powell, J. R. \& Badley, R. A. Analysis of bacterial function by multi-colour fluorescence flow cytometry and single cell sorting. J. Microbiol. Methods 42, 97-114 (2000).

24 Tamayo, M., Santiso, R., Gosalvez, J., Bou, G. \& Fernandez, J. L. Rapid assessment of the effect of ciprofloxacin on chromosomal DNA from Escherichia coli using an in situ DNA fragmentation assay. BMC Microbiol. 9, 69 (2009).

25 Hawkey, P. M. Mechanisms of quinolone action and microbial response. J. Antimicrob. Chemother. 51(Suppl 1), 29-35 (2003).

26 Drlica, K., Engle, E. C. \& Manes, S. H. DNA gyrase on the bacterial chromosome: possibility of two levels of action. Proc. Natl Acad. Sci. USA 77, 6879-6883 (1980).

27 Fernandez, J L et al. DNA fragmentation in microorganisms assessed in situ. Appl. Environ. Microbiol. 74, 5925-5933 (2008).

28 Walberg, M., Gaustad, P. \& Steen, H. B. Rapid assessment of ceftazidime, ciprofloxacin, and gentamicin susceptibility in exponentially-growing $E$. coli cells by means of flow cytometry. Cytometry 27, 169-178 (1997).

29 Johansson, L. \& Liden, G. A study of long-term effects on plasmid-containing Escherichia coli in carbon-limited chemostat using 2D-fluorescence spectrofluorimetry. Biotechnol. Prog. 22, 1132-1139 (2006). 